\title{
Marginal Fermi Liquid in Twisted Bilayer Graphene
}

\author{
J. González ${ }^{1}$ and T. Stauber ${ }^{2}$ \\ ${ }^{1}$ Instituto de Estructura de la Materia, CSIC, E-28006 Madrid, Spain \\ ${ }^{2}$ Materials Science Factory, Instituto de Ciencia de Materiales de Madrid, CSIC, E-28049 Madrid, Spain
}

(Received 10 May 2019; revised manuscript received 19 September 2019; accepted 17 April 2020; published 8 May 2020)

\begin{abstract}
Linear resistivity at low temperatures is a prominent feature of high- $T_{c}$ superconductors which has also been found recently in twisted bilayer graphene. We show that due to an extended van Hove singularity (VHS), the $T$-linear resistivity can be obtained from a microscopic tight-binding model for filling factors close to the VHS. The linear behavior is shown to be related to the linear energy dependence of the electron quasiparticle decay rate which implies the low-energy logarithmic attenuation of the quasiparticle weight. These are distinctive features of a marginal Fermi liquid, which we also see reflected in the respective lowtemperature logarithmic corrections of the heat capacity and the thermal conductivity, leading to the consequent violation of the Wiedemann-Franz law. We also show that there is a crossover at $T \sim 6 \mathrm{~K}$ from the marginal Fermi liquid regime to a regime dominated by excitations on the Dirac cone right above the VHS that also yields a linear resistivity albeit with smaller slope, in agreement with experimental observations.
\end{abstract}

DOI: $10.1103 /$ PhysRevLett.124.186801

Introduction.-The discovery of a correlated insulating [1] and superconducting [2] state in magic angle twisted bilayer graphene (TBG) has stimulated great interest, both from the theoretical [3-27] as well as from the experimental side [28-34]. One striking and astonishing result of the initial experiments was the similarity of the phase diagram to the one of high- $T_{c}$ superconductors [35-38]. This analogy has further been manifested by the observation of a strange metal regime with its linear temperature dependence of the resistivity [39,40].

The nature of the strange metal phase in TBG is highly controversial. It is frequently assumed that electron-phonon interactions could be responsible for the anomalous behavior of the resistivity, but at the same time acknowledging that phonons cannot account for the $T$-linear dependence down to the lowest temperatures reached in the experiments $(\sim 0.5 \mathrm{~K})$, in particular below the Debye frequency scale (in the case of optical phonons) or below the Bloch-Grüneisen temperature (in the case of acoustic phonons). The proposal derived in this Letter solves this puzzle, presenting a consistent explanation that is purely based on electronelectron interaction.

Our key observation is that the lowest-energy bands of TBG near the magic angle display two distinct features that dominate the transport properties. These are the Dirac nodes at the charge neutrality point and, on the other hand, a set of extended saddle points which, according to experimental $[41,42]$ and also theoretical $[43,44]$ evidence, are close to the Fermi level at half-filling of the two highest (lowest) valence (conduction) bands. The extended saddle points are characterized by a dispersion with very small curvature along the $\Gamma K$ lines that manifests in the straight segments of the Fermi line for the second highest valence band (VB) represented in Fig. 1(b). We have argued that this feature could be at the origin of the observed superconductivity of TBG, relying on a Kohn-Luttinger mechanism where the strongly anisotropic screening leads to an attractive interaction between Cooper pairs around the Fermi line [45].

In this Letter, we show that the decay of the low-energy quasiparticles in the region of flat dispersion [typically

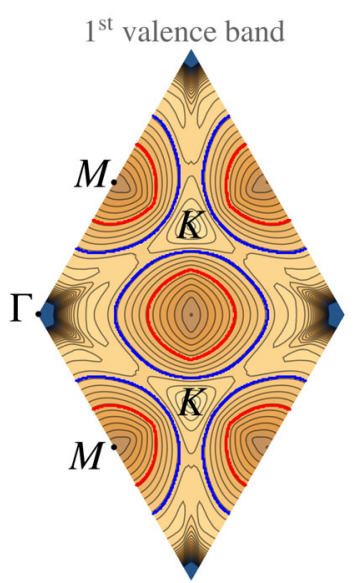

(a)

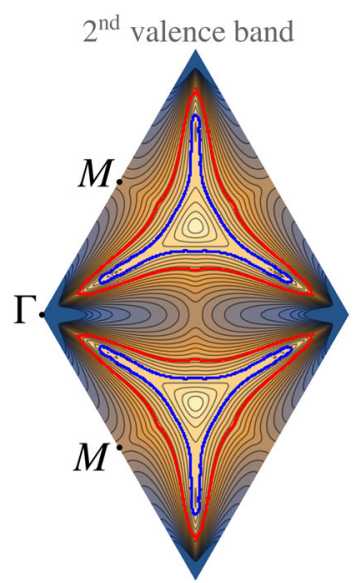

(b)
FIG. 1. Energy contour maps (with light colors representing higher energies) of the first and the second highest valence band in the moiré Brillouin zone of a twisted graphene bilayer with twist angle $\theta_{28} \approx 1.16^{\circ}$, showing the Fermi lines for filling levels shifted -0.2 (blue lines) and $-1.5 \mathrm{meV}$ (red lines) below the level of the saddle points placed along the $\Gamma K$ lines. 
within $1 \mathrm{meV}$ around the extended van Hove singularity (VHS)] as well as of those with higher energy (already located in the Dirac cone below the $K$ point) can account for a $T$-linear dependence of the resistivity. The origin of the $T$-linear behavior is, however, different in the two regimes, forcing the appearance of a crossover regime, i.e., a change of slope of the resistivity around a crossover temperature $T$. This change in the slope is, indeed, observed in the measurements reported in Ref. [39].

Furthermore, the $T$-linear resistivity observed in the lowtemperature regime below $\sim 5 \mathrm{~K}$ must be just one of the many facets revealing the deviation of TBG from the conventional Fermi liquid picture. The reason for such an anomalous behavior lies in the linear growth with energy of particle-hole excitations across the straight segments of the blue Fermi line shown in Fig. 1(b). This kind of linear scaling was actually the main assumption in the original proposal of the marginal Fermi liquid paradigm [46], developed phenomenologically to describe the normal phase of the high- $T_{c}$ superconductors. Our derivation, therefore, can be seen as a concrete realization of the paradigm, which should manifest itself in other observable features of TBG such as the linear energy scaling of the quasiparticle decay rate or the anomalous temperature dependence of the heat capacity and the thermal conductivity.

Model.-To model TBG, we will use the commensurable tight-binding model parametrized by the integer $i$ corresponding to the twist angle with $\cos \theta_{i}=\left[\left(3 i^{2}+3 i+0.5\right) /\right.$ $\left.\left(3 i^{2}+3 i+1\right)\right][47,48]$. The hopping parameters are taken from Refs. [49,50] such that the nearest-neighbor intralayer hopping is set to $t=-2.7 \mathrm{eV}$ and the vertical interlayer hopping to $t_{\perp}=0.48 \mathrm{eV}$. For a recent review on bilayer systems, see Ref. [51] and also the Supplemental Material [52].

We note that the anomalous transport behavior arises from the peculiar features of the second highest VB of TBG. For small twist angle $\theta \approx 1^{\circ}$, the first and second highest VBs have coincident VHS at the same energy, but with very different dispersion in the two cases. This dispersion takes the form of a conventional saddle point in the first VB, while that in the second VB has a more distorted shape, with a much smaller curvature along the $\Gamma K$ line than in the orthogonal direction.

In Fig. 1, the energy contour maps of the two highest VBs are shown for a bilayer with twist angle $\theta_{28} \approx 1.16^{\circ}$. Also shown are the Fermi lines for two energies $\Delta \mu$ relative to the VHS. In the second highest VB, they consist of two patches with three lobes each. Notable are the straight segments with quasi-one-dimensional dispersion in the second highest $\mathrm{VB}$ for $\Delta \mu=-0.2 \mathrm{meV}$, which are a reflection of the extended VHS.

Let us also stress that the energy contour maps of Fig. 1 with their characteristic Fermi lines around the VHS appear to be rather universal, i.e., independent of the specific details of the underlying microscopic model that may include relaxation effects or slightly different hopping parameters. Remarkably, the same extended VHS are also found in the continuum model [54-57]; see Ref. [45].

Resistivity.-We compute the resistivity relying on the semiclassical formalism of the Boltzmann equation. In this approximation and assuming an on-site Hubbard interaction $U$, the resistivity $\rho_{n}$ in the direction of the unit vector $n$ can be expressed as an average over momenta $\boldsymbol{k}$ [58]

$$
\rho_{\boldsymbol{n}}=\rho_{0} \frac{\frac{1}{T} \int \frac{d^{2} k}{(2 \pi)^{2}} \frac{n_{F}\left(\varepsilon_{\boldsymbol{k}}\right)}{\tau_{\mathrm{tr}}(\boldsymbol{k})}}{\left(\int \frac{d^{2} k}{(2 \pi)^{2}} \frac{\partial n_{F}\left(\varepsilon_{\boldsymbol{k}}\right)}{\partial \varepsilon_{k}}\left(\boldsymbol{v}_{\boldsymbol{k}} \cdot \boldsymbol{n}\right)^{2}\right)^{2}},
$$

where $\rho_{0}=h / e^{2}$ (restoring for a moment Planck's constant), $n_{F}\left(\varepsilon_{\boldsymbol{k}}\right)$ is the Fermi-Dirac distribution function at energy $\varepsilon_{k}$, the Fermi velocity is $v_{k}=\nabla \varepsilon_{k}$, and $1 / \tau_{\text {tr }}$ stands for the transport decay rate. At this point, it is instructive to discern the different contributions from the decay of quasiparticles in the $i$ th $\mathrm{VB}$ to the $j$ th VB, which lead to partial decay rates

$$
\begin{aligned}
\frac{1}{\tau_{\mathrm{tr}}^{(i j)}(\boldsymbol{k})}= & U^{2} \int \frac{d^{2} k^{\prime}}{(2 \pi)^{2}} \int_{0}^{\varepsilon_{k}^{(i)}} d \omega\left|\left\langle i, \boldsymbol{k} \mid j, \boldsymbol{k}^{\prime}\right\rangle\right|^{2} \\
& \times\left[1-n_{F}\left(\varepsilon_{\boldsymbol{k}^{\prime}}^{(j)}\right)\right] \delta\left(\varepsilon_{\boldsymbol{k}}^{(i)}-\varepsilon_{\boldsymbol{k}^{\prime}}^{(j)}-\omega\right) \operatorname{Im} \chi_{\mathrm{tr}}^{(i j)}\left(\boldsymbol{k}, \boldsymbol{k}^{\prime} ; \omega\right),
\end{aligned}
$$

with the imaginary part of the transport susceptibility

$$
\begin{aligned}
& \operatorname{Im} \chi_{\operatorname{tr}}^{(i j)}\left(\boldsymbol{k}, \boldsymbol{k}^{\prime} ; \omega\right) \\
& =\int \frac{d^{2} p}{(2 \pi)^{2}}\left|\left\langle l, \mathbf{p} \mid l^{\prime}, \mathbf{p}+\boldsymbol{k}-\boldsymbol{k}^{\prime}\right\rangle\right|^{2} n_{F}\left(\varepsilon_{\mathbf{p}}^{(l)}\right)\left[1-n_{F}\left(\varepsilon_{\mathbf{p}+\boldsymbol{k}-\boldsymbol{k}^{\prime}}^{\left(l^{\prime}\right)}\right)\right] \\
& \quad \times\left[\left(\boldsymbol{v}_{\boldsymbol{k}}^{(i)}+\boldsymbol{v}_{\mathbf{p}}^{(l)}-\boldsymbol{v}_{\boldsymbol{k}^{\prime}}^{(j)}-\boldsymbol{v}_{\mathbf{p}+\boldsymbol{k}-\boldsymbol{k}^{\prime}}^{\left(l^{\prime}\right)} \cdot \boldsymbol{n}\right]^{2} \delta\left(\varepsilon_{\mathbf{p}+\boldsymbol{k}-\boldsymbol{k}^{\prime}}^{\left(l^{\prime}\right)}-\varepsilon_{\mathbf{p}}^{(l)}-\omega\right) .\right.
\end{aligned}
$$

Above, we have introduced the eigenvectors $|i, \boldsymbol{k}\rangle$ corresponding to states in the $i$ th highest VB with eigenenergies $\varepsilon_{\boldsymbol{k}}^{(i)}$, and the sum over $l, l^{\prime}$ is implied [59]. The Hubbard coupling $U$ is the projected on-site interaction onto the second highest VB of the moiré lattice, which we set to $U / 2 \pi=3 \mathrm{meV} a_{M}^{2}, a_{M}$ being the lattice constant of the superlattice.

The behavior of the resistivity and the transport scattering rate depends on the shift $\Delta \mu$ of the Fermi level with respect to the extended saddle points shown in Fig. 1. When the Fermi level is close to the VHS (with a deviation $\Delta \mu$ within $\lesssim 0.5 \mathrm{meV}$ ), the low-energy electron quasiparticles have a dominant decay channel into particle-hole excitations across the straight segments of the blue Fermi line shown in Fig. 1(b). These excitations have an approximate linear energy-momentum dispersion, realizing then one of the basic assumptions of the marginal Fermi liquid paradigm. We stress that such a decay channel acts efficiently 


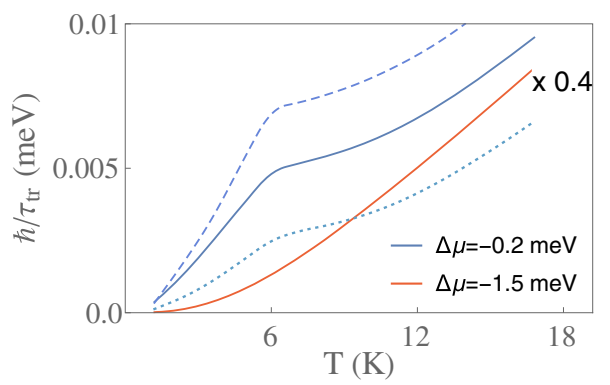

FIG. 2. Plot of the temperature dependence of the values averaged over the Fermi line (and weighted with the inverse of the square of the Fermi velocity to get dimensions of energy) of $1 / \tau_{\mathrm{tr}}^{(11)}$ (dashed line), $1 / \tau_{\mathrm{tr}}^{(22)}$ (solid line), and $1 / \tau_{\mathrm{tr}}^{(12)}$ (dotted line), when the Fermi level is 0.2 (blue curves) and $1.5 \mathrm{meV}$ (red curve, scaled by a factor of 0.4 ) below the VHS.

for the intraband scattering of quasiparticles in both the first and the second highest VB. Consequently, the temperature dependence of $1 / \tau_{\mathrm{tr}}^{(11)}$ and $1 / \tau_{\mathrm{tr}}^{(22)}$ for these low-energy quasiparticles (with $\varepsilon_{\boldsymbol{k}}$ within $\sim 0.5 \mathrm{meV}$ around the VHS) is found to be linear in the two bands, as can be seen in Fig. 2. Moreover, interband scattering is shown to provide only a subdominant, softly quadratic correction given by $1 / \tau_{\mathrm{tr}}^{(12)}$, represented also in Fig. 2.

Beyond a certain temperature, however, the quasiparticles are excited at higher energies away from the VHS, lying on the Dirac cone which is right above the extended saddle points. These quasiparticles can decay into particlehole excitations not attached to the straight segments of the Fermi line, providing a conventional scattering mechanism that is reflected in the departure from $T$-linear behavior above $T \sim 6 \mathrm{~K}$ in the plot of Fig. 2 .

We, therefore, see that the extended saddle-point regime leads to a crossover in the temperature dependence of the transport decay rate, whenever the shift $\Delta \mu$ of the Fermi level is sufficiently small. On the other hand, if the Fermi level significantly departs from the VHS (with $|\Delta \mu| \gtrsim 1 \mathrm{meV}$ ) then the Fermi line recovers a more regular (two-dimensional) shape, as shown by the red lines in Fig. 1, and the scattering of electron quasiparticles follows a conventional trend. In this case, the temperature dependence of the transport decay rate has a quadratic behavior starting at low $T$, as shown in Fig. 2.

From the results for the transport decay rate, we obtain the resistivity by applying Eq. (1). At low temperatures, we may assume that only quasiparticles in the energy range of $T$ contribute, so that the resistivity can be computed as an average over the branches of the Fermi line in the two highest VBs. We may discern again different contributions $\rho_{i j}$ from the intraband and interband scattering processes accounted for by $1 / \tau_{\mathrm{tr}}^{(i j)}$. Decomposing the momentum $\boldsymbol{k}$ into a longitudinal $k_{\|}$ and a transverse $k_{\perp}$ component, we have

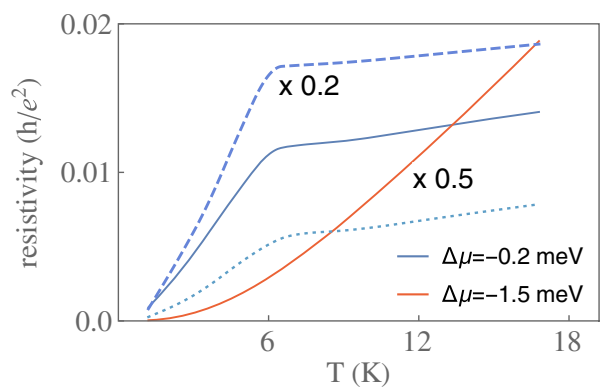

FIG. 3. Plot of the temperature dependence of $\rho_{11}$ (dashed line, scaled by a factor of 0.2$), \rho_{22}$ (solid line), and $\rho_{12}$ (dotted line), for a shift of the Fermi level $\Delta \mu=-0.2$ (blue curves) and $-1.5 \mathrm{meV}$ (red curve, scaled by a factor of 0.5 ) with respect to the level of the VHS.

$$
\rho_{i j} \sim \rho_{0} \frac{1}{T} \oint_{\mathcal{C}_{i}} d k_{\|} \int \frac{d \varepsilon_{\boldsymbol{k}}^{(i)}}{v_{\boldsymbol{k}}^{(i)}} \frac{n_{F}\left(\varepsilon_{\boldsymbol{k}}^{(i)}\right)}{\tau_{\mathrm{tr}}^{(i j)}(\boldsymbol{k})},
$$

$\mathcal{C}_{i}$ being the Fermi line of the corresponding VB.

In Fig. 3, one can see that the crossover and the $T$-linear behavior at low temperature of $1 / \tau_{\mathrm{tr}}^{(11)}$ and $1 / \tau_{\mathrm{tr}}^{(22)}$ are translated into a similar behavior of the resistivities $\rho_{11}$ and $\rho_{22}$. Interband scattering is found to provide only a subdominant, softly quadratic correction $\rho_{12}$. Above the crossover temperature, however, Eq. (4) introduces an additional $T$ dependence, due to the fact that the quasiparticles decaying from the Dirac cone have a reduction of phase space as the energy increases towards the Dirac node at the $K$ point. The $1 / T$ factor in Eq. (4) is thus not canceled and the temperature dependence of the resistivity becomes approximately linear above the crossover temperature, as shown in Fig. 3.

We note that the magnitude of the resistivity $\rho_{11}$ turns out to be larger than the one originating from the second highest VB. This is a consequence of the fact that the effective bandwidth (for momenta close to the Fermi line) is larger in this latter band. The electronic transport will thus be short circuited through this second highest VB and it is this band that will dictate the dominant behavior of the resistivity.

As was the case for the transport decay rate, the behavior of the resistivity crucially depends on the shift of the Fermi level with respect to the VHS. This is evidenced in the quadratic behavior of the red curve in Fig. 3 that refers to $\Delta \mu=-1.5 \mathrm{meV}$. Nevertheless, for $\Delta \mu=-0.2 \mathrm{meV}$, the linear $T$ dependence of the resistivity is quite clear, although with different slope above and below a crossover temperature of the order of $\sim 6 \mathrm{~K}$. Very suggestively, a change in the slope of the resistivity has also been seen in the experimental observations around half filling of the moiré unit cell, displaying a larger slope of the linear $T$ dependence below a crossover temperature that is slightly above $5 \mathrm{~K}$ in the measurements reported in Ref. [39]. For more details, see the Supplemental Material [52]. 


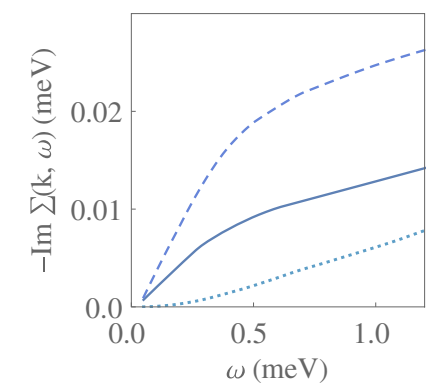

(a)

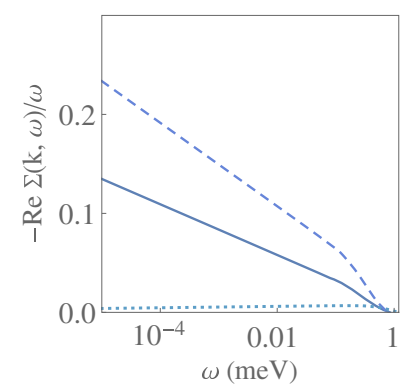

(b)
FIG. 4. Plot of the frequency dependence of the real and the imaginary part of the values averaged over the Fermi line of $\Sigma^{(11)}$ (dashed line), $\Sigma^{(22)}$ (solid line), and $\Sigma^{(12)}$ (dotted line), for a shift of the Fermi level $\Delta \mu=-0.2 \mathrm{meV}$ below the VHS.

Quasiparticle properties.-The linear low-temperature dependence of the transport decay rate can be related to the low-energy behavior of the electron self-energy $\Sigma(\boldsymbol{k}, \omega)$. When analyzing this quantity, it is convenient to discern the different contributions to its imaginary part from the decay of quasiparticles in the $i$ th VB to the $j$ th VB. These can be computed in terms of the conventional electron-hole susceptibility $\chi(\boldsymbol{q}, \omega)$ as

$$
\begin{aligned}
\operatorname{Im} \Sigma^{(i j)}(\boldsymbol{k}, \omega)= & -U^{2} \int \frac{d^{2} p}{(2 \pi)^{2}} \int_{-\infty}^{\infty} d \omega_{p}|\langle i, \boldsymbol{k} \mid j, \mathbf{p}\rangle|^{2} \\
& \times \operatorname{sgn}\left(\omega_{p}\right) \delta\left(\omega_{p}-\varepsilon_{\mathbf{p}}^{(j)}\right) \operatorname{Im} \chi\left(\boldsymbol{k}-\mathbf{p}, \omega-\omega_{p}\right) .
\end{aligned}
$$

The real part of the self-energy can be obtained by making use of the Kramers-Kronig relation, which in this case takes the form

$$
\operatorname{Re} \Sigma(\boldsymbol{k}, \omega)=\frac{2 \omega}{\pi} \int_{0}^{\infty} d \Omega \frac{\operatorname{Im} \Sigma(\boldsymbol{k}, \Omega)}{\Omega^{2}-\omega^{2}} .
$$

When the Fermi level is close to the VHS, the imaginary part of $\Sigma^{(11)}$ and $\Sigma^{(22)}$ computed from Eq. (5) has a linear dependence on the frequency $\omega$ which is similar to the lowtemperature dependence of $1 / \tau_{\mathrm{tr}}^{(11)}$ and $1 / \tau_{\mathrm{tr}}^{(22)}$, as can be seen in Fig. 4(a). As shown in the same figure, the effect of interband scattering is reflected in a small correction from $\Sigma^{(12)}$ at low frequencies. Consequently, the dependence of the real part of the self-energy on $\omega$ displays a significant deviation from linear behavior, with a logarithmic correction which is another evidence of the departure from Fermi liquid behavior. This is shown in Fig. 4(b), which represents the average over the Fermi line of the real part of the different contributions $\Sigma^{(i j)}(\boldsymbol{k}, \omega) / \omega$ when the Fermi level is shifted by $\Delta \mu=-0.2 \mathrm{meV}$ with respect to the VHS.

We observe that the real part of the dominant intraband contributions to the self-energy behaves as $\Sigma(\boldsymbol{k}, \omega) \sim$ $\omega \log (\omega)$ at low frequencies, which implies that the electron quasiparticles are progressively attenuated when approaching the Fermi level. The dressed electron propagator becomes

$$
G(\boldsymbol{k}, \omega)=\frac{1}{\omega-\varepsilon_{\boldsymbol{k}}-\Sigma(\boldsymbol{k}, \omega)} \sim \frac{\Delta}{\omega-\varepsilon_{\boldsymbol{k}}+i \gamma \omega}
$$

after rewriting the self-energy corrections in terms of the quasiparticle weight $\Delta$ and the imaginary shift $i \gamma \omega$ of the quasiparticle pole. The quasiparticle weight is suppressed following the low-energy scaling $\Delta \sim 1 /|\log (\omega)|$, which is the hallmark of the marginal Fermi liquid behavior $[46,60]$. For more details, see the Supplemental Material [52].

Heat capacity and thermal conductivity.-The anomalous behavior of the electron quasiparticles has also a significant impact on the temperature dependence of observables like the heat capacity. This is obtained from the entropy $S$, which can be expressed as an integral along the Fermi line by decomposing again the momentum $\boldsymbol{k}$ into longitudinal $k_{\|}$and transverse $k_{\perp}$ components $[52,61]$ :

$\frac{S}{A} \approx \frac{1}{2 \pi^{2}} \frac{1}{T} \oint \frac{d k_{\|}}{v_{\boldsymbol{k}}} \int_{-\infty}^{\infty} d \omega \omega \frac{\partial n_{F}(\omega)}{\partial \omega}[\omega-\operatorname{Re} \Sigma(\boldsymbol{k}, \omega)]$,

$A$ being the area of the system. Then, by absorbing the temperature $T$ into a dimensionless variable $\omega / T$ in the integrand of Eq. (8), we see that the anomalous scaling of the electron self-energy translates into the dominant scaling behavior $S \sim T|\log (T)|$.

The heat capacity $C$ is obtained by taking the derivative of $S$ with respect to $T$ and it inherits, therefore, the logarithmic correction that we find in the entropy:

$$
C=T \frac{\partial}{\partial T} \frac{S}{A} \sim T|\log (T)|
$$

We see therefore that the logarithmic correction to the heat capacity holds in the same range of anomalous behavior of the self-energy plotted in Fig. 4, which corresponds in temperature to the range $T \lesssim 10 \mathrm{~K}$.

The logarithmic correction of the heat capacity has also a direct translation into the temperature dependence of the thermal conductivity $\kappa$. This quantity is related to the heat capacity through the thermal diffusivity $\alpha$ according to the formula $\kappa=\alpha C$. The thermal diffusivity is in turn proportional to the mean free path of the energy carriers [62]. When the Fermi level is close to the VHS, we can apply the linear low-temperature dependence we have found in the transport scattering rate to estimate the mean free path [46]. This implies that

$$
\kappa(T)=\alpha C \sim|\log (T)| .
$$

This anomalous scaling should be observable down to the temperature scale at which the transport starts to be dominated by the scattering from disorder (impurities or 
lattice defects) in the twisted bilayer. Above that scale, the ratio between the thermal conductivity and the electrical conductivity should be also affected by the logarithmic correction from Eq. (10), thus leading to a modification of the Wiedemann-Franz law [46].

Long-range interaction.-So far, we have considered the case of a strongly screened Hubbard interaction that can be interpreted as some effective parameter $U$ that also includes the dielectric constant of the substrate. Within the continuum model [54] we have further investigated the relaxation time for long-range interaction including screening effects [63] that come from the top and back gate as well as from internal self-screening. Interestingly, apart from the linear vs quadratic behavior as a function of the chemical potential relative to the VHS, we obtain relaxation times comparable to the Planckian limit for gate distances $D=15 \mathrm{~nm}$; see Fig. 4 of the Supplemental Material [52]. Within the same framework, we have discussed the influence of the relaxation time to the quasilocalized plasmonic modes [64], which also leads to a $T$-linear behavior proportional to the density of states, see Supplemental Material [52].

Summary.-Relying on a tight-binding approach, we have been able to obtain a linear temperature dependence of the resistivity for filling factors around the VHS in the two highest VBs of TBG, in the framework of a model with onsite Hubbard interaction $U$. At low temperatures, the linear behavior of the resistivity can be traced back to the more general frequency dependence of the electron Green's function, characterized by a logarithmic correction indicating marginal Fermi liquid behavior. We thus predict that fingerprints of a marginal Fermi liquid should also be present in the heat capacity and the thermal conductivity. Observing these features experimentally may be a way to discriminate between the electron-electron and the electron-phonon interaction as the possible driving force for the superconducting state as well as for the unconventional normal state found near half-filling of the two highest VBs in TBG.

Finally, we stress that the scaling laws we have discussed persist when the Coulomb interaction is extended to get a finite spatial range. In that case, quantitative predictions about the different observables may be greatly enhanced and, specially in the limit of a long-range Coulomb interaction (with appropriate internal screening), a regime of nearly Planckian resistivity can be reached, with the transport decay rate approaching the bound given by $T / \hbar$.

This work has been supported by Spain's MINECO under Grant No. FIS2017-82260-P as well as by the CSIC Research Platform on Quantum Technologies PTI-001.

[1] Y. Cao, V. Fatemi, A. Demir, S. Fang, S. L. Tomarken, J. Y. Luo, J. D. Sanchez-Yamagishi, K. Watanabe, T. Taniguchi, E. Kaxiras, R. C. Ashoori, and P. Jarillo-Herrero, Nature (London) 556, 80 (2018).
[2] Y. Cao, V. Fatemi, S. Fang, K. Watanabe, T. Taniguchi, E. Kaxiras, and P. Jarillo-Herrero, Nature (London) 556, 43 (2018).

[3] C. Xu and L. Balents, Phys. Rev. Lett. 121, 087001 (2018).

[4] G. E. Volovik, JETP Lett. 107, 516 (2018).

[5] N. F. Q. Yuan and L. Fu, Phys. Rev. B 98, 045103 (2018).

[6] H. C. Po, L. Zou, T. Senthil, and A. Vishwanath, Phys. Rev. B 99, 195455 (2019).

[7] B. Roy and V. Juričić, Phys. Rev. B 99, 121407(R) (2019).

[8] H. Guo, X. Zhu, S. Feng, and R. T. Scalettar, Phys. Rev. B 97, 235453 (2018).

[9] J. F. Dodaro, S. A. Kivelson, Y. Schattner, X. Q. Sun, and C. Wang, Phys. Rev. B 98, 075154 (2018).

[10] G. Baskaran, arXiv:1804.00627.

[11] C.-C. Liu, L.-D. Zhang, W.-Q. Chen, and F. Yang, Phys. Rev. Lett. 121, 217001 (2018).

[12] K. Slagle and Y. B. Kim, SciPost Phys. 6, 16 (2019).

[13] T. J. Peltonen, R. Ojajärvi, and T. T. Heikkilä, Phys. Rev. B 98, 220504(R) (2018).

[14] D. M. Kennes, J. Lischner, and C. Karrasch, Phys. Rev. B 98, 241407(R) (2018).

[15] M. Koshino, N. F. Q. Yuan, T. Koretsune, M. Ochi, K. Kuroki, and L. Fu, Phys. Rev. X 8, 031087 (2018).

[16] J. Kang and O. Vafek, Phys. Rev. Lett. 122, 246401 (2019).

[17] H. Isobe, N. F. Q. Yuan, and L. Fu, Phys. Rev. X 8, 041041 (2018).

[18] Y.-Z. You and A. Vishwanath, npj Quantum Mater. 4, 16 (2019).

[19] F. Wu, A. H. MacDonald, and I. Martin, Phys. Rev. Lett. 121, 257001 (2018).

[20] Y.-H. Zhang, D. Mao, Y. Cao, P. Jarillo-Herrero, and T. Senthil, Phys. Rev. B 99, 075127 (2019).

[21] J. M. Pizarro, M. J. Calderón, and E. Bascones, J. Phys. Commun. 3, 035024 (2019).

[22] H. K. Pal, arXiv:1805.08803.

[23] M. Ochi, M. Koshino, and K. Kuroki, Phys. Rev. B 98, 081102(R) (2018).

[24] A. Thomson, S. Chatterjee, S. Sachdev, and M. S. Scheurer, Phys. Rev. B 98, 075109 (2018).

[25] S. Carr, S. Fang, P. Jarillo-Herrero, and E. Kaxiras, Phys. Rev. B 98, 085144 (2018).

[26] F. Guinea and N. R. Walet, Proc. Natl. Acad. Sci. U.S.A. 115, 13174 (2018).

[27] L. Zou, H. C. Po, A. Vishwanath, and T. Senthil, Phys. Rev. B 98, 085435 (2018).

[28] M. Yankowitz, S. Chen, H. Polshyn, Y. Zhang, K. Watanabe, T. Taniguchi, D. Graf, A. F. Young, and C. R. Dean, Science 363, 1059 (2019).

[29] Y. Choi, J. Kemmer, Y. Peng, A. Thomson, H. Arora, R. Polski, Y. Zhang, H. Ren, J. Alicea, G. Refael, F. von Oppen, K. Watanabe, T. Taniguchi, and S. Nadj-Perge, Nat. Phys. 15, 1174 (2019).

[30] A. L. Sharpe, E. J. Fox, A. W. Barnard, J. Finney, K. Watanabe, T. Taniguchi, M. A. Kastner, and D. GoldhaberGordon, Science 365, 605 (2019).

[31] S. Moriyama, Y. Morita, K. Komatsu, K. Endo, T. Iwasaki, S. Nakaharai, Y. Noguchi, Y. Wakayama, E. Watanabe, D. Tsuya, K. Watanabe, and T. Taniguchi, arXiv:1901.09356.

[32] Y. Jiang, X. Lai, K. Watanabe, T. Taniguchi, K. Haule, J. Mao, and E. Y. Andrei, Nature (London) 573, 91 (2019). 
[33] Y. Xie, B. Lian, B. Jäck, X. Liu, C.-L. Chiu, K. Watanabe, T. Taniguchi, B. A. Bernevig, and A. Yazdani, Nature (London) 572, 101 (2019).

[34] A. Kerelsky, L. J. McGilly, D. M. Kennes, L. Xian, M. Yankowitz, S. Chen, K. Watanabe, T. Taniguchi, J. Hone, C. Dean, A. Rubio, and A. N. Pasupathy, Nature (London) 572, 95 (2019).

[35] R. Micnas, J. Ranninger, and S. Robaszkiewicz, Rev. Mod. Phys. 62, 113 (1990).

[36] A. Damascelli, Z. Hussain, and Z.-X. Shen, Rev. Mod. Phys. 75, 473 (2003).

[37] P. A. Lee, N. Nagaosa, and X.-G. Wen, Rev. Mod. Phys. 78, 17 (2006).

[38] G. R. Stewart, Rev. Mod. Phys. 83, 1589 (2011).

[39] Y. Cao, D. Chowdhury, D. Rodan-Legrain, O. RubiesBigorda, K. Watanabe, T. Taniguchi, T. Senthil, and P. Jarillo-Herrero, Phys. Rev. Lett. 124, 076801 (2020).

[40] H. Polshyn, M. Yankowitz, S. Chen, Y. Zhang, K. Watanabe, T. Taniguchi, C. R. Dean, and A. F. Young, Nat. Phys. 15, 1011 (2019).

[41] Y. Kim, P. Herlinger, P. Moon, M. Koshino, T. Taniguchi, K. Watanabe, and J. H. Smet, Nano Lett. 16, 5053 (2016).

[42] Y. Cao, J. Y. Luo, V. Fatemi, S. Fang, J. D. SanchezYamagishi, K. Watanabe, T. Taniguchi, E. Kaxiras, and P. Jarillo-Herrero, Phys. Rev. Lett. 117, 116804 (2016).

[43] T. Cea, N. R. Walet, and F. Guinea, Phys. Rev. B 100, 205113 (2019).

[44] L. Rademaker, D. A. Abanin, and P. Mellado, Phys. Rev. B 100, 205114 (2019).

[45] J. González and T. Stauber, Phys. Rev. Lett. 122, 026801 (2019).

[46] C. M. Varma, P. B. Littlewood, S. Schmitt-Rink, E. Abrahams, and A. E. Ruckenstein, Phys. Rev. Lett. 63, 1996 (1989).

[47] E. Suárez Morell, J. D. Correa, P. Vargas, M. Pacheco, and Z. Barticevic, Phys. Rev. B 82, 121407(R) (2010).
[48] G. T. de Laissardière, D. Mayou, and L. Magaud, Nano Lett. 10, 804 (2010).

[49] I. Brihuega, P. Mallet, H. González-Herrero, G. Trambly de Laissardière, M. M. Ugeda, L. Magaud, J. M. GómezRodríguez, F. Ynduráin, and J.-Y. Veuillen, Phys. Rev. Lett. 109, 196802 (2012).

[50] P. Moon and M. Koshino, Phys. Rev. B 87, 205404 (2013).

[51] A. Rozhkov, A. Sboychakov, and A. Rakhmanov, and F. Nori, Phys. Rep. 648, 1 (2016).

[52] See Supplemental Material at http://link.aps.org/ supplemental/10.1103/PhysRevLett.124.186801 for more details and additional numerical results, which includes Ref. [53].

[53] T. Stauber, T. Low, and G. Gómez-Santos, Phys. Rev. B 98, 195414 (2018).

[54] J. M. B. L. dos Santos, N. M. R. Peres, and A. H. Castro Neto, Phys. Rev. Lett. 99, 256802 (2007).

[55] E. J. Mele, Phys. Rev. B 81, 161405(R) (2010).

[56] R. Bistritzer and A. H. MacDonald, Proc. Natl. Acad. Sci. U.S.A. 108, 12233 (2011).

[57] P. Moon and M. Koshino, Phys. Rev. B 85, 195458 (2012).

[58] R. Hlubina and T. M. Rice, Phys. Rev. B 51, 9253 (1995).

[59] In practice, we confine the sum in Eq. (3) to intraband processes in the second VB, which is justified as these give rise to the dominant susceptibility arising from electron-hole excitations across the straight segments of the Fermi line in Fig. 1(b).

[60] P. B. Littlewood and C. M. Varma, J. Appl. Phys. 69, 4979 (1991).

[61] A. A. Abrikosov, L. P. Gorkov, and I. E. Dzyaloshinski, Methods of Quantum Field Theory in Statistical Physics (Dover, New York, 1963).

[62] J. M. Ziman, Principles of the Theory of Solids (Cambridge University Press, Cambridge, England, 1972).

[63] G. F. Giuliani and J. J. Quinn, Phys. Rev. B 26, 4421 (1982).

[64] T. Stauber and H. Kohler, Nano Lett. 16, 6844 (2016). 